\title{
Discussion of Load Combination Parameters for Heavy-cargo Transportation in Passing Highway Bridge
}

\author{
Ping Lu 1, a , Yulin Zhou ${ }^{1, ~ c}$, Haiyang $\mathrm{Yu}^{2}{ }^{2}$, , Haijun $\mathrm{Wu}{ }^{1, \mathrm{~d}}$ \\ ${ }^{1}$ School of Civil Engineering, Chongqing Jiaotong University, Chongqing, China. \\ ${ }^{2}$ Construction Engineering Quality Supervision Station of Jiangjin District of Chongqing, China. \\ a58392137@qq.com, b352276628@qq.com, c1253617647@qq.com, d583921237@qq.com
}

\begin{abstract}
Keywords: bridge engineering; highway heavy-cargo transportation; load combination coefficient; transverse allocation; dynamic programming

Abstract: To discuss about choosing the parameters of bearing capacity with over-size transport passing the bridge, this study analyzes the characteristics of designing load of heavy transportation which are different from the general cars; according to the characteristics of accidental load of heavy-cargo transportation, this research analyzes and proposes the load combination principle of heavy-cargo transportation load; discussions and recommendations have been made on the method of determining load bearing partial coefficients for calculation of over-size transport of highway bridges.
\end{abstract}

\section{Introduction}

With the development of China's industrial and transportation modernization, the extra-large and extra-heavy industrial equipment on expressways has been increasing. Heavy-cargo transportation and overload transportation increase the load on bridges, a bridge to speed up the rate of injury and damage. Most of the equipment for over-size transportation is related to the people's livelihood. Therefore, it is particularly important to ensure the safety of bridges and cargo during heavy transportation of highways. In view of the super-load in the load size, load distribution, frequency of large parts, access control conditions and many other aspects, there are obvious differences from the general vehicle load law. Thus, it is of great practical significance to study the relevant parameters for calculation and analysis of bridge bearing capacity under heavy-cargo transportation.

\section{The basic characteristics of heavy-cargo transportation}

Heavy-cargo transportation load is a temporary, accidental special load for the load standard used in the design of expressway bridges, with low frequency of occurrence and short duration of action; for a specific super-load, its total weight, axle weight, wheelbase, track and other parameters are relatively determined values. Thus, how to use the bearing capacity of bridge in an appropriate way is a problem that needs to be solved when heavy-cargo transportation of bridge is analyzed. Unlike general road vehicle transport, heavy-cargo transportation generally has the following salient features [1]:

(1) Certain mandatory heavy-cargo transportation

Most of the goods transported by over-size are the key equipment for national key investment projects. To ensure that the project is completed and put into operation on time, over-size transport vehicles must pass through as scheduled. Thus heavy-cargo transportation has certain mandatory.

(2) Low emerging frequency of heavy-cargo transportation

Compared with the general vehicles driving on the road, the probability and frequency of occurrence of over-size transport vehicles are much smaller than those of ordinary vehicles. Therefore, compared with the vehicle load corresponding to the bridge design load, the repeated structural effects and fatigue effects caused by the bridge are much smaller.

(3) Heavy-cargo transportation could pass through the bridge after declaration and approval of competent authority

General cargo vehicles can straightway pass through the bridge without approval of competent authority. However, due to the fact that over-size transport vehicles have characteristics such as 
super-load, they must apply to the competent department of traffic before they travel. Without the approval of regulatory agencies highway over-size transport vehicles may not travel on the roads.

(4) Heavy-cargo transportation must follow the time and route specified by the approval agency

With resulting in significant effecting on the traffic situation, over-size transport are requested to run avoiding peak-hour traffic by approval agency, thereby furthest reducing the effecting on the traffic situation. At the same time, due to the characteristics of cargoes and transportation destinations, the approval department often has identified the best time and the best route for transportation when issuing passes. Meanwhile, the traffic police department and the road administration department will generally adopt security measures such as closed traffic and police car escort to ensure the safety of goods and traffic operations along the route. Thus, heavy-cargo transportation is generally under strict supervision and the timing of transit and the specific location can be adjusted and controlled.

(5) Over-size transport vehicles are strictly limited in speed when running

In order to ensure the safety of large cargoes themselves, vehicles and transportation facilities, the State has strict regulations on the speed of heavy-cargo transportation. Generally, on a flat road the speed does not exceed $20 \mathrm{~km} / \mathrm{h}$, the speed at which the vehicles meet is not more than $8 \mathrm{~km} / \mathrm{h}$ and when passing mountain roads and bridges, the speed limit in $5 \mathrm{~km} / \mathrm{h}$ or less. Meanwhile, sudden braking and sudden acceleration should be avoided throughout the entire transportation process.

(6) Heavy-cargo transportation often does not work with other vehicles on the bridge

Highway bridge structure is designed based on the investigation of the state of the vehicle load on the road and design load standards proposed by the vehicle development are properly considered through probabilistic methods. Over-size transport vehicles (including cargos) have a large total weight and far exceed the design specifications. For this reason, when over-size vehicles crossing the bridge, to ensure the maximum safety of highway bridge structures, the traffic department would adopt measures, such as running on axis lined and prohibiting other vehicles and pedestrians.

\section{The load combination principle of heavy-cargo transportation load}

Article 4.1.6 of the "General Code for Design of Highway Bridges and Culverts" (JTG D60-2004) [2] designs the limit state of the bearing capacity of highway bridges and culverts and divides them into two action effect combinations according to their possible effects, namely basic combination and accidental combination. The basic combination of action effect refers to the combination of the design value effect of permanent action and the variable action design value effect, this combination is used in the conventional design of the structure, is all highway bridge structure should be considered. The accidental combination of action effect refers to the effect combination of the permanent action standard value, the representative value of variable action and an accidental action standard value. The partial effect coefficient of the accidental action is 1.0 , and the variable action that occurs at the same time as the accidental action depends on its specific conditions, regardless of its participation in the combination. For the calculation of the limit state of the bearing capacity of a vehicle that may exceed the "Limits of Dimensions, Axle Load and Masses for Road Vehicles" (GB1589-2004) [3] on a highway, the partial coefficient may be taken as 1.1.

Heavy-cargo transportation load is a temporary and accidental load for load standard of highway bridge design and bridge design is based on the most unfavorable design of the loading control [4]. According to the analysis of the first part of this paper, we can know that the way of super-load can be strictly limited, so that it can be adopted in the most favorable way to maximize the excavation and utilization of the structural resistance of the bridge.

Over-size transport vehicles, heavier than general overload vehicles, are required to pass through in the optimum circumstance of temperature load and on the optimum position along the transverse of the bridge, simultaneously, other vehicles and pedestrians are prohibited. Therefore, permanent load and super-load only be considered in checking bearing capacity without other variable actions. 


\section{Partial coefficients of load combination in heavy-cargo transportation load}

Judging whether over-size transport vehicles could safely cross the bridge with the failure probability of bridge structure under one certain important failure mode and use structural reliability theory to quantify the safety of the heavy transportation crossing the bridge. The use of probability statistics to deal with the uncertainty of load and structural resistance and its impact on the structural reliability, scientific and objective to make a reasonable assessment of the safety bridge.

According to the failure criterion and failure mode of bridge structure, load probability model, structure resistance probability model and reliability theory knowledge in "Research on Reliability Evaluation of Concrete Girder Bridges under Super Vehicle Loads" [5], consulting the method of using the limit state design of bridge structure with partial coefficient, limit state equation with partial coefficient can be established for judging the safety of over-size transport vehicles when passing through the bridge. The equations are as follows:

$$
\begin{aligned}
& R_{K} \geq S_{K} . \\
& R_{K}=K_{3} K_{4} K_{5}\left(K_{1} S_{G K}+K_{2} S_{Q K}\right) . \\
& S_{K}=\gamma_{0} \gamma_{1} \gamma_{2}\left(1.2 S_{G}+\gamma_{T} S_{T}\right) .
\end{aligned}
$$

According to the principle that the difference between the structural resistance standard value obtained after the expression of the partial coefficient and the reliability index of the predetermined target are the minimum, partial coefficient is drawn up and first order second moment method is used to find the component coefficients corresponding to the target reliability. Under these conditions of the structural resistance, permanent loads effect, loads effect of overweight vehicles and other parameters by statistical data in article [5], by means of given incipient partial coefficient of overweight vehicles $\gamma_{\mathrm{T}}=1.00,1.02,1.04,1.08,1.10,1.12,1.14$ and given the ratio of loads effect of overweight vehicles to permanent loads effect $\rho=0.1,0.2,0.4,0.8,1.0,1.5,2.0,2.5,3.0$. Reliability calculation shows: the calculated reliability index, $\beta$, of the flexural member under each partial coefficient is higher, the reliability index value when the partial coefficient is 1.08 is higher than the target reliability standard value which is ruled in the specification and the partial coefficient of oversize vehicles of $\gamma_{\mathrm{T}}=1.08$. According to "Research on Load Control Standards of Large-scale Transport on Medium and Small Span PC Simply Supported Girder Bridge" [6], the partial coefficient of heavy-cargo transportation load has been researched by considering having an impact on the structural resistance by bridge technique condition and using the value which reduce the reliability index of the norm by 0.25 ; by giving the incipient partial coefficient of heavy-cargo transportation load $\gamma_{\mathrm{T}}=1.00,1.02,1.04,1.08,1.10,1.12,1.14$ and the ratio of heavy-cargo transportation load effect to permanent load effect $\rho=0.1,0.2,0.4,0.8,1.0,1.5,2.0,2.5,3.0$, under the condition that partial coefficient of permanent and resistance coefficient are kept consistent in norm, the bridges of different classification are checked on reliability. According to the conclusions, the partial coefficients of heavy-cargo transportation load are assigned the value 1.0 with grade-one bridge in technical condition, 1.06 with grade-two and 1.12 with grade-three.

Considering that technical conditions of the highway bridges are basically all belong to the grade-one and grade-two bridge, mainly grade-two. Due to the differences in means of inspection and level of understanding, technical condition and the bearing capacity of the bridge are not absolutely corresponding, additionally heavy-cargo transportation load belonging to accidental load which essentially is the same as the load of trailer in "General Code for Design of Highway Bridges and Culverts" (JTJ 021-85) with tiny variability. In the perspective of facilitating the structural analysis, the partial coefficient of heavy-cargo transportation load that can be approximated by safety is 1.1 .

At the same time, the special traffic control conditions for over-size transport vehicles passing the bridge should be considered in the calculation, such as the prohibition of accelerating and braking, 
passing through with uniform speed $5 \mathrm{~km} / \mathrm{h}$, driving more to the roads' center and so on. Therefore, the vehicle impact coefficient may not be considered when calculating.

\section{Conclusions}

This paper discusses the selection of calculation parameters in the evaluation process of bearing capacity when over-size transport vehicles passing through the bridge, as the following conclusions:

(1) Compared with the general vehicle load, heavy-cargo transportation load is a temporary and relatively accidental special load with low frequency and short duration. The over-size transport vehicle is generally under strict supervision and the time of its passage and the specific location of the load can be adjusted and controlled. So that supervision and control make the selection of calculation parameters for heavy-cargo transportation load to be different from the general design calculation.

(2) Over-size transport vehicles, heavier than general overload vehicles, are required to pass through in the optimum circumstance of temperature load and on the optimum position along the transverse of the bridge, meanwhile, other vehicles and pedestrians are prohibited. Thus, permanent load and super-load only be considered in checking bearing capacity without other variable actions.

(3) Considering technical conditions of highway bridges are all belong to grade-one and grade-two bridge, mainly grade-two, additionally heavy-cargo transportation load belonging to accidental load which essentially is the same as the load of trailer in "General Code for Design of Highway Bridges and Culverts" (JTJ 021-85) with tiny variability. In the view of facilitating the structural analysis, the partial coefficient of heavy-cargo transportation load that can be approximated by safety is 1.1 .

\section{Acknowledgements}

This paper is partially supported by the Fundamental Research Funds for the Central Universities (No.310821161120), Chongqing Science and Technology Commission Social Livelihood Project (cstc2017shmsA30022), Traffic Science and Technology Project of Chongqing Highway Bureau "Study and Application of Applicable Safety Monitoring System and Assessment Technology for Medium-sized Bridges" and Scientific Projects of State Key Laboratory Breeding Base of Mountain Bridge and Tunnel Engineering (CQSLBF-Y12-5, CQSLBE-Y14-12, CQSLBF-Y15-17).

\section{References}

[1] Haiyang Yu. Study on the Large-cargo Transportation Analysis Methods and Control Standard of Conventional Bridge on Chongqing Highway[D]. Chongqing: Chongqing Jiaotong University, 2014.

[2] Ministry of Communications of the People's Republic of China. JTG D60-2004. General Code for Design of Highway Bridges and Culverts[S]. Beijing: China Communications Press, 2004.

[3] General Administration of Quality Supervision, Inspection and Quarantine of the People's Republic of China, Standardization Administration of the People's Republic of China. GB1589-2004. Limits of Dimensions, Axle Load and Masses for Road Vehicles [S]. Beijing: China Communications Press, 2004.

[4] Xuefeng Liu. The Research on Load Carrying Capacity of Bridge When Conducting Large Parts Transportation[J]. Shanxi Architecture, 2009, 35(36): 328-329.

[5] Aide He. Research on Reliability Evaluation of Concrete Girder Bridges under Super Vehicle Loads[D]. Guangdong: Guangdong University of Technology, 2011.

[6] Zhibin Li. Research on Load Control Standards of Large-scale Transport on Medium and Small Span PC Simply Supported Girder Bridge[D]. Chongqing: Chongqing Jiaotong University, 2012. 